\title{
Mastopatia Diabética como diagnóstico diferencial para neoplasia de mama: revisão integrativa da literatura
}

\author{
Diabetic Mastopathy as a differential diagnosis for breast cancer: integrative literature review \\ Mastopatía diabética como diagnóstico diferencial del cáncer de mama: revisión integradora de la \\ literatura
}

Recebido: 18/10/2021 | Revisado: 25/10/2021 | Aceito: 29/10/2021 | Publicado: 01/11/2021

\author{
Gabriela Troncoso \\ ORCID: https://orcid.org/0000-0003-3483-679X \\ Universidade de Patos de Minas, Brasil \\ E-mail: gabrielatroncoso@unipam.edu.br \\ Álvaro Alves de Sá Júnior \\ ORCID: https://orcid.org/0000-0003-1706-8450 \\ Universidade Federal de Jataí, Brasil \\ E-mail: alvaroalve1@gmail.com \\ Letícia Dias Escoura \\ ORCID: https://orcid.org/0000-0001-5889-2601 \\ Universidade de Uberaba, Brasil \\ E-mail: leticiaescoura@gmail.com \\ Rafaela Melo Sisconetto \\ ORCID: https://orcid.org/0000-0002-5952-4939 \\ Universidade de Uberaba, Brasil \\ E-mail: rafaelasisconetto@gmail.com \\ Douglas Reis Abdalla \\ ORCID: https://orcid.org/0000-0002-6971-1201 \\ Universidade de Uberaba e Cursos de Saúde, Brasil \\ Faculdade de Talentos Humanos, Brasil \\ E-mail: drabdalla@facthus.edu.br
}

\begin{abstract}
Resumo
Introdução: Mastopatia Diabética é uma doença rara e benigna que afeta as mamas, sendo um dos principais diagnósticos diferenciais relacionados aos carcinomas mamários. Entretanto, apesar de apresentar características de imagem e clínicas similares a processos malignos de mama, foi evidenciado que tal patologia não apresenta relação com evoluções pré-malignas ou malignas. Tal patologia parece estar relacionada com uma resposta autoimune secundária a um estado hiperglicêmico prolongado, presente em pacientes diabéticos. Objetivo: buscar na literatura evidências acerca da mastopatia diabética como manifestação diferencial às lesões neoplásicas da mama. Metodologia: Revisão Integrativa da literatura na base de dados PubMed das produções de 2008 a 2021. Os descritores utilizados foram: "mastopatia diabética"; "mastopatia"; "diabetes mellitus"; "mama"; "patologias da mama", os quais foram conjugados para delimitação da busca. Resultados: A pesquisa realizada encontrou 45 estudos, dentre os quais foram selecionados 13 que contemplavam a pergunta norteadora. A revisão revelou que a patologia retratada apresenta características que requerem uma análise cuidadosa do histórico clínico com exame físico e complementação com exames de imagem. A biópsia, sobretudo por agulha grossa, é o método diagnóstico mais eficiente para elucidação histopatológica. Além disso, devido à crescente presença dos fatores de risco para o desenvolvimento dessa doença na população atual, espera-se que a mastopatia diabética se torne mais comum. Considerações finais: A mastopatia diabética é, portanto, um importante diagnóstico diferencial para neoplasias de mama cujo diagnóstico é bastante complexo e exige, para além dos aspectos clínicos, a análise de componentes radiológicos e laboratoriais.
\end{abstract}

Palavras-chave: Diabetes mellitus; Carcinoma mamário; Complicações do diabetes.

\begin{abstract}
Introduction: Diabetic Mastopathy is a rare and benign disease that affects the breasts, being one of the main differential diagnoses related to breast carcinomas. However, despite presenting imaging and clinical features similar to malignant breast processes, it was evidenced that this pathology is not related to premalignant or malignant evolutions. This pathology seems to be related to an autoimmune response secondary to a prolonged hyperglycemic state, present in diabetic patients. Objective: To search the literature for evidence of diabetic mastopathy as a differential manifestation of neoplastic breast lesions. Methodology: Integrative literature review on the PubMed
\end{abstract}


database of productions from 2008 to 2021. The descriptors used were: "diabetic mastopathy"; "mastopathy"; "diabetes mellitus"; "breast"; "breast pathologies", which were conjugated to delimit the search. Results: The search found 45 studies, from which 13 were selected that contemplated the guiding question. The review revealed that the pathology portrayed presents characteristics that require a careful analysis of the clinical history with physical examination and complementation with imaging exams. Biopsy, especially by thick needle, is the most efficient diagnostic method for histopathological elucidation. Furthermore, due to the increasing presence of risk factors for the development of this disease in the current population, diabetic mastopathy is expected to become more common. Final considerations: Diabetic mastopathy is, therefore, an important differential diagnosis for breast neoplasms whose diagnosis is quite complex and requires, besides clinical aspects, the analysis of radiological and laboratory components.

Keywords: Diabetes mellitus; Breast carcinoma; Diabetes complications.

\begin{abstract}
Resumen
Introducción: La mastopatia diabética es una enfermedad rara y benigna que afecta a las madres, siendo uno de los principales diagnósticos relacionados con los carcinomas de mama. Sin embargo, a pesar de presentar características clínicas y de imagen similares a los procesos mamarios malignos, se ha evidenciado que esta patología no está relacionada con evoluciones premalignas o malignas. Dicha patología parece estar relacionada con una respuesta autoinmune secundaria a un estado hiperglucémico prolongado, presente en los pacientes diabéticos. Objetivo: buscar en la literatura evidencias sobre la mastopatia diabética como manifestación diferencial a las lesiones neoplásicas de la mama. Metodología: Revisión bibliográfica integradora en la base de datos PubMed de producciones desde 2008 hasta 2021. Los descriptores utilizados fueron: "mastopatía diabética"; "mastopatía"; "diabetes mellitus"; "mama"; "patologías mamarias", que se conjugaron para delimitar la búsqueda. Resultados: En la búsqueda realizada se encontraron 45 estudios, entre los que se seleccionaron 13 que contemplaban la pregunta guía. La revisión reveló que la patología retratada presenta características que requieren un análisis cuidadoso de la historia clínica con el examen físico y la complementación con los exámenes de imagen. La biopsia, especialmente con aguja gruesa, es el método de diagnóstico más eficaz para el esclarecimiento histopatológico. Además, debido a la creciente presencia de factores de riesgo para el desarrollo de esta enfermedad en la población actual, se espera que la mastopatía diabética sea más común. Consideraciones finales: La mastopatía diabética es, por tanto, un importante diagnóstico diferencial de las neoplasias de mama cuyo diagnóstico es bastante complejo y requiere, además de los aspectos clínicos, el análisis de los componentes radiológicos y de laboratorio.
\end{abstract}

Palabras clave: Diabetes mellitus; Carcinoma de mama; Complicaciones de la diabetes.

\title{
1. Introdução
}

No ano de 1984, Soler e Khardori relataram pela primeira vez a Mastopatia Diabética (MD), também conhecida como Mastopatia Linfocítica, a qual é uma doença rara e benigna que afeta as mamas, sendo um dos principais diagnósticos diferenciais relacionados aos carcinomas mamários. Entretanto, apesar de apresentar características de imagem e clínicas similares a processos malignos de mama, foi evidenciado que tal patologia não apresenta relação com evoluções pré-malignas ou malignas (Alkhudairi, et al; 2020).

A MD é uma condição clínica que representa menos de $1 \%$ das lesões benignas da mama, sendo mais frequente em mulheres no período de pré-menopausa que possuem diabetes mellitus (DM) tipo 1 insulino-dependentes desde a época juvenil, apresentando incidência de $0,6 \%$ a $13 \%$. Entretanto, já foi relatado em mulheres com DM tipo 2, em não diabéticas, em portadoras de doenças autoimunes como tireoidite de Hashimoto, Lúpus Eritematoso Sistêmico (LES) e, em menores proporções, em homens. (Francisco, et al; 2012) Nesse sentido, o uso do termo para pacientes não diabéticos ainda está em discussão, já que existe a denominação "mastopatia linfocítica", mas a maioria dos autores ainda faz uso de "mastopatia diabética" (Agochukwu \&Wong, 2017).

Ainda não há uma compreensão total quanto a sua etiopatogenia, porém teorias sugerem que possa ser multifatorial. Dentre as principais hipóteses, alguns autores afirmam que essa patologia se trata de uma resposta autoimune secundária a um estado hiperglicêmico prolongado. A hiperglicemia induz a glicosilação, aumentando a ligação cruzada intermolecular, alterando a expansão da matriz em quantidade e qualidade, ficando resistente à degradação. Assim, esse acúmulo anormal na matriz e os produtos glicosilados atuam como neoantígenos e desencadeiam uma resposta autoimune com células B, havendo 
produção e proliferação de autoanticorpos. Há uma mediação realizada por macrófagos, os quais liberam citocinas que podem atuar como fatores de crescimento, permitindo a expansão da matriz e proliferação de colágeno (Neetu et al., 2010).

No entanto, à medida que essa hipótese é sustentada inclusive pelos estudos de Soler e Khardori devido ao padrão de infiltrado linfocítico de células $\mathrm{B}$, essa teoria ainda é refutada por alguns por não explicar essa condição em pacientes não portadores de DM. Ademais, um padrão semelhante de inflamação é visto em outras doenças autoimunes, incluindo artrite reumatoide, doença da tireóide, anemia perniciosa e Sjogrens, para as quais existem relatos de coexistência em pacientes diabéticos afetados por MD (Neetu et al.,2010, Pereira et al., 2010, Dorkhova et al., 2012).

Apresenta-se clinicamente pelo aparecimento de massa palpáveis nas mamas, uni ou bilateralmente, móveis, de consistência firme ou dura, indolores, solitárias ou múltiplas e irregulares (Agochukwu \& Wong, 2017). Embora não seja comum, dor e desconforto nas mamas podem estar presentes também, de modo a afetar a realização de atividades diárias (Perret et al., 2006). Além disso, pode-se encontrar outras complicações decorrentes da diabetes, como neuropatia, nefropatia e retinopatia diabéticas, sendo esta última a mais comum; bem como algumas síndromes autoimunes, principalmente em indivíduos não diabéticos que apresentaram mastopatia linfocítica (Tomaszewski et al., 1992, Leroux-Stewart \& RabasaLhoret, 2014, Agochukwu \& Wong, 2017).

O diagnóstico é pautado em alguns pilares: história clínica; exame físico; exames de imagem, como ultrassonografia, ressonância magnética e mamografia; estudo histopatológico e métodos de biópsia, como punção aspirativa por agulha fina (PAAF) e core biopsy (biópsia de agulha grossa guiada por ultrassom ou mamografia) (Machado \& Regalo; 2020). Esse processo é difícil, visto que as características encontradas são semelhantes aos carcinomas de mama, sendo essencial uma pesquisa minuciosa e completa (Rajasundaram et al; 2020).

A história clínica desses pacientes envolve queixas recentes e muitas vezes recorrentes de processo inflamatório nas mamas, associado a diabetes mellitus insulino-dependente de longa evolução e de mau controle (Loscos et al; 2021). No exame físico, verifica-se massas palpáveis unilaterais ou bilaterais, de consistência firme, móvel e geralmente indolor (Gupta et al., 2019). Os exames de imagem, principalmente a mamografia, revelam achados como parênquima denso com densidades assimétricas e presença de tecido glandular denso e difuso, inicialmente sugestivos de malignidade (Leroux \& Rabasa, 2019).

Os métodos de biópsia como a PAAF e a core biopsy, são essenciais para a visualização histopatológica do tecido mamário e diagnóstico (Shrikrishnapalasuriyar et al, 2018). Os critérios histológicos para a Mastopatia Diabética foram estabelecidos em 1994 por Seidman et al., sendo eles: (1) estroma com fibras colágenas com características semelhantes a quelóide e com uma concentração aumentada de células fusiformes e (2) linfócitos maduros agrupados em torno de pequenos vasos sanguíneos, lóbulos e ductos.

Não existe tratamento específico para a MD, de modo que o recomendado é apenas o acompanhamento clínico e de imagem (Leroux-Stewart et al, 2014). Em alguns casos, em que há dor e desconforto nas mamas, pode ser realizada a mastectomia com preservação dos mamilos com uma possível reconstrução imediata bilateral com implantes (Agochukwu et al, 2017). Salvo os casos com apresentação de dor e desconforto, a mastectomia não é indicada, uma vez que as lesões podem recorrer de forma mais extensiva após a ressecção (Leroux-Stewart et al, 2014). A lumpectomia para cada uma das lesões, em casos de nódulos múltiplos nas mamas, não é recomendada, uma vez que configura um procedimento tedioso tanto para o cirurgião quanto para a paciente, além do risco de recorrência das lesões após ressecção pelo mesmo princípio que ocorre na mastectomia (Agochukwu et al, 2017).

Desta forma, a presente revisão integrativa visa buscar na literatura evidências acerca da mastopatia diabética como manifestação diferencial às lesões neoplásicas da mama. 


\section{Metodologia}

No presente estudo foi conduzida uma revisão integrativa, que consiste em uma pesquisa que permite a partir de evidências a avaliação, síntese e conhecimento acerca de um fenômeno, objetivando produzir uma visão geral de conceitos complexos, teorias ou problemas de saúde relevantes a partir de estudos pré-existentes, possibilitando a proposição de intervenção (Casarin et al, 2020).

Para a seleção dos artigos, foram conduzidas 6 etapas metodológicas, quais sejam: 1. elaboração da questão norteadora ou hipótese da pesquisa, ou seja, identificou-se o problema, apresentou-se o mecanismo de busca e os descritores ou palavras chave; 2. estabelecimento dos critérios de inclusão e exclusão dos artigos a serem selecionados para composição da amostra; 3. leitura exploratória dos títulos e resumos dos artigos para pré-seleção; 4. leitura analítica dos artigos a fim de compilar, analisar e categorizar as informações; 5. interpretação dos resultados. 6. síntese seguida da apresentação dos resultados identificados, que permeiam a questão norteadora (De Sousa et al., 2011).

Portanto, neste estudo optou-se por realizar busca sobre os conceitos: mastopatia diabética; diabetes mellitus e patologias da mama. A partir desses conceitos, definiu-se a questão norteadora: seria a mastopatia diabética um diagnóstico diferencial para o câncer de mama analisando seus aspectos clínicos, laboratoriais e radiológicos?.

Após a formulação da questão a ser pesquisada, foi realizado um levantamento bibliográfico na plataforma PubMed. O levantamento do estudo ocorreu entre abril e junho de 2021. E a seleção dos textos procedeu com as buscas na plataforma, utilizando os filtros nela disponíveis para textos publicados entre 2009 e 2021. Para seleção das publicações, foram adotados os seguintes critérios de inclusão: artigos científicos, publicados no idioma Inglês, entre os anos de 2009 a 2021, disponíveis online e gratuitamente na íntegra. Foram excluídos os artigos sem resumo na base de dados ou incompletos, editoriais, cartas ao editor, estudos reflexivos, revisões sistemáticas ou integrativas de literatura.

Após a definição da questão norteadora, localização e seleção dos artigos, foram identificadas 45 publicações potencialmente elegíveis para serem incluídas nessa revisão. Após a aplicação dos critérios de inclusão e exclusão a amostra foi composta por 15 publicações, foram analisados os resumos de 15 registros, para verificar se atenderiam os critérios de elegibilidade e se responderiam à pergunta que norteia esta revisão, assim excluiu-se 02 registros e somente 13 foram analisados na íntegra para confirmar a elegibilidade para a síntese quantitativa e análise dos dados conforme o fluxograma de seleção (Figura 1). 
Figura 1. Fluxograma de Seleção das Publicações.

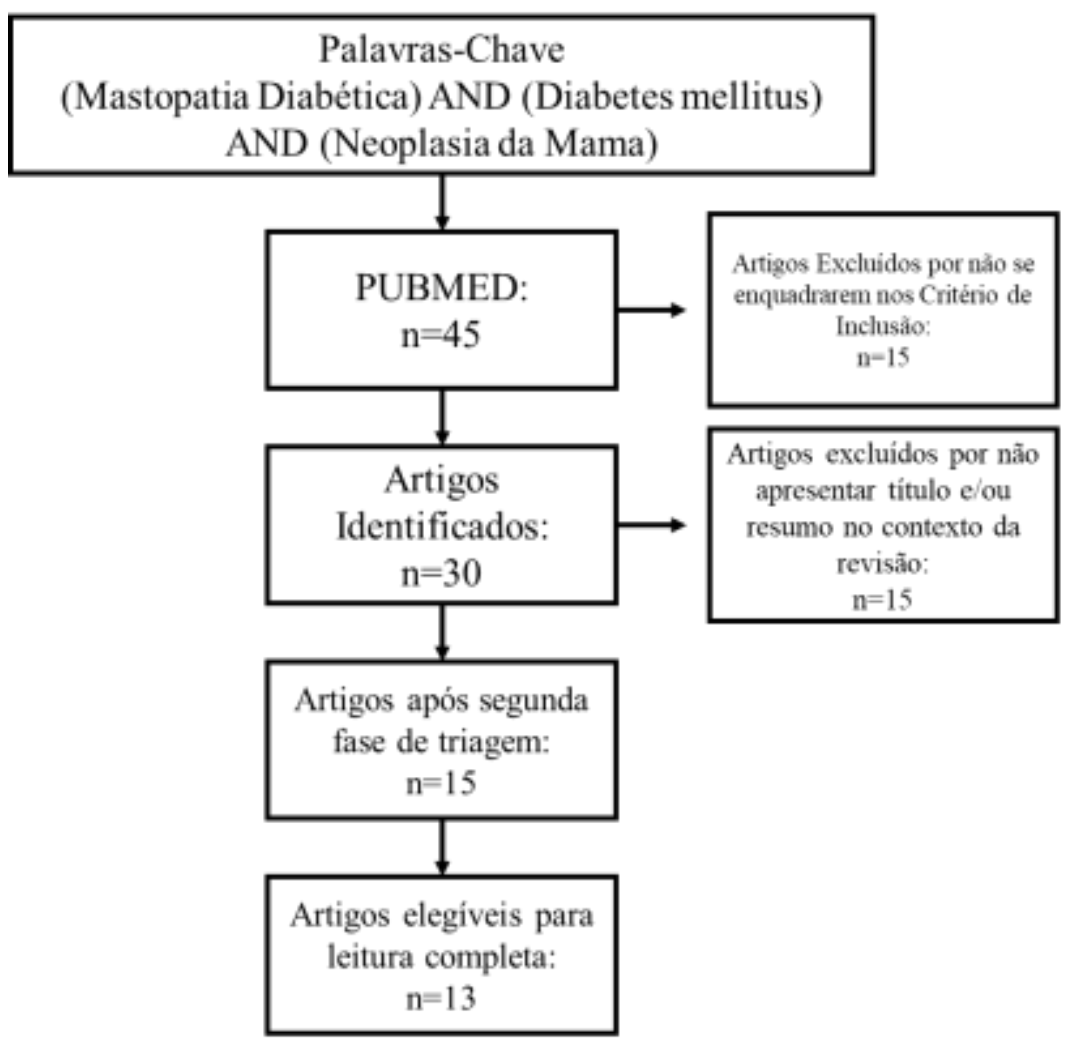

Fonte: Autores (2021).

\section{Resultados e Discussão}

No espaço de tempo delimitado para a realização deste estudo (2009-2021) foram encontradas e analisadas 13 publicações, sumarizadas na Tabela 1. Em 2009, 2011, 2016, 2019 e 2020 foram publicados 1 artigos (7,69\%) em cada ano, respectivamente. No ano de 2012 constam 5 publicações (38,47\%). Já no ano de 2014 foram publicados 3 (23,08\%) artigos, respectivamente.

De acordo com a metodologia dos trabalhos selecionados, 12 deles são relatos de caso, enquanto que apenas 1 corresponde a um estudo retrospectivo, conforme sumarizado no Tabela 1. 
Tabela 1. Artigos levantados nas bases de dados Pubmed, sobre mastopatia diabética.

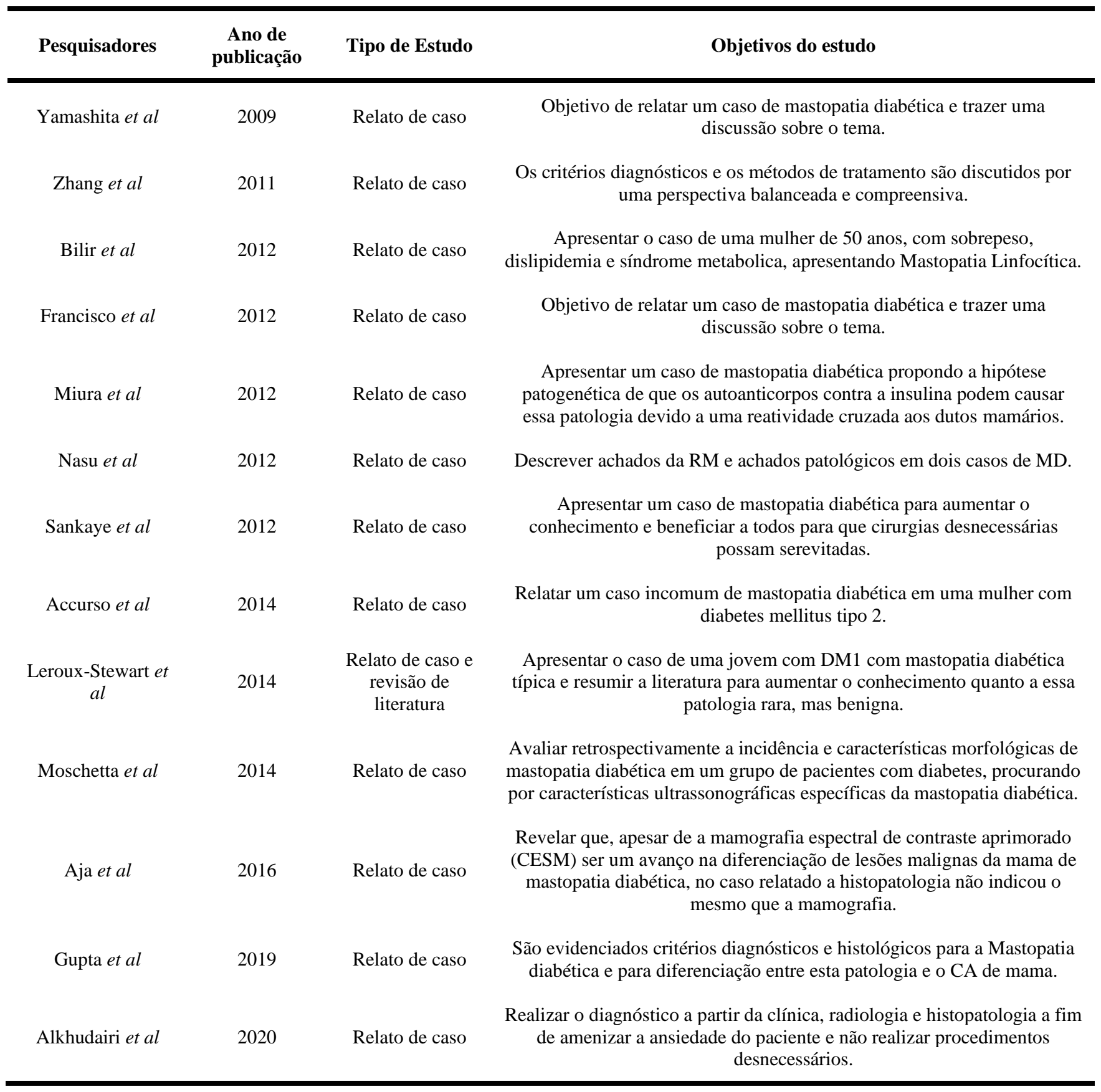

Fonte: Autores (2021).

As publicações resultaram de diferentes revistas sendo:Clinical Breast Cancer; Canadian Journal of Diabetes; Australasian Medical Journal; Case Reports in Medicine; Breast Care; The Japanese Breast Cancer Society (2); The American Journal of Medicine; Wiley Periodicals; Journal of Medical Imaging and Radiation Oncology; Clinical Imaging; International Journal of Surgery; Cureus.

Analisando os locais de estudo 3 artigos foram realizados no Japão (23,08\%),2 artigos desenvolvidos na Itália $(15,38 \%)$ e em cada um desses países, sendo eles China, Índia, Arábia Saudita, Turquia, Canadá, Estados Unidos, Espanha e Portugal, foram publicados um artigo respectivamente $(7,69 \%)$.

Na pesquisa de Yamashita et al. (2009), abordou-se caso de suspeita de MD que incluia diagnóstico de carcinoma de mama: sexo feminino, 64 anos, pós-menopausa, história de DM tipo 2 não controlada e insulino-dependente há 18 anos e com 
retinopatia diabética, negou histórico de tumores malignos na família. Em mamografia de rotina, apresentou calcificações em mama esquerda, mas no exame físico não havia massa palpável e sim uma mama de parênquima denso. Ultrassom (US) indicou massa hipoecoica irregular com sombra acústica, de $11 \mathrm{~mm}$ de diâmetro em quadrante superior lateral esquerdo, além de biópsia com agulha guiada por US que mostrou fibrose hialina sem evidências de malignidade. Ademais, Ressonância Magnética (RM) mostoru lesão em quadrante superior em região medial na mama esquerda, não podendo distinguir de câncer de mama. Então, foi necessária uma biópsia a vácuo, na qual a histopatologia apontou carcinoma ductal não invasivo. Em seguida, foi feita uma quadrantectomia junto a um linfonodo sentinela, os quais o estudo anatomo-patológico indicou fibrose, fibroblastos epitelioides, vasculite e lobulite linfocítica e ductite, caracterizando lesão típica de MD, entretanto, haviam células tumorais difusas com calcificação ductal de carcinoma in situ, mas linfonodo sentinela descartou metástases. Foi definido como diagnóstico final carcinoma ductal invasivo acompanhado de MD em mama esquerda, sem alterações em mama direita. O diagnóstico precoce de carcinoma de mama é dificultado quando a paciente tem histórico sugestivo de MD. Em contrapartida com os demais artigos, este sugere que mesmo que MD seja diagnosticada, seja feito um acompanhamento por meio de mamografia, US e RM e, se qualquer microcalcificação ou área focal alterada for observada, realizar biópsia por agulha fina a fim de realizar um diagnóstico precoce.

No trabalho de Zhang et al. (2011), buscou-se discutir critérios diagnósticos e métodos de tratamento para a MD, a partir de duas pacientes. Paciente 1, sexo feminino, 56 anos, pós-menopausa, com história de diabetes há 38 anos, há 1 ano com administração de Glargina $14 \mathrm{U}$, apresentou ao exame físico massas únicas bilaterais em mamas com um ano de desenvolvimento. Paciente 2, 50 anos, pós-menopausa, com história de diabetes mellitus há 4 anos, em uso de Novolin 30R, 25U, 2x/dia, apresentou massas únicas bilaterais em mamas há 6 meses. Como resultados da Paciente 1, mamografia indicou achados assimétricos, moderadamente densos, de alta densidade no quadrante superior esquerdo de mama direita, múltiplas calcificações bilaterais e com linfonodos axilares palpáveis e densos a exame físico. Já em US de mama, duas massas de áreas unidas, hipoecóicas, irregulares e mal definidas em quadrante superior esquerdo da mama direita, além do doppler indicando hemorragia pontual em ambas massas e lesão hipoecóica no quadrante superior medial da mama esquerda, sem sinal de fluxo sanguíneo. Decidiram realizar biópsia excisional, na qual foi encontrado Esclerose Linfocítica Lobular, indicando um sinal de inflamação no tecido mamário, e pequenas áreas com presença de células parecidas com Linfócito B. Já na Paciente 2, sua US evidenciou manifestação usual de MD, com massa única na mama esquerda (posição lateral de 3 horas) e múltiplas massas na mama direita (lateralmente às 9 horas e 12 horas no quadrante superior), com mama lipossubstituída, sem sinais de fluxo sanguíneo detectados. Na mamografia apresentou sombreamento de alta densidade no quadrante superior lateral da mama direita sem sinal de malignidade. Também foi indicada a realização de biópsia excisional, a qual confirmou MD, com presença de receptores CD3, CD20 e Ki-67, sendo sugerido acompanhamento longitudinal. A partir disso, reforça que a MD é uma doença fibrótica benigna incomum que ocorre nas mamas e de difícil diferenciação com o câncer de mama tanto pelas manifestações clínicas como achados de imagem. Deve-se atentar ao fato de que a MD está relacionada aos pacientes com DM1 e que, geralmente, é acompanhada de complicações sistêmicas da doença, como retinopatia diabética, e são insulinodependentes.

Segundo Bilir et al. (2012), apesar de a mastopatia linfocítica ser mais relacionada com doenças autoimunes, principalmente em pacientes com DM, a doença pode ocorrer também em outros pacientes com síndrome metabólica. Em seu trabalho, foi apresentado o caso de uma mulher de 50 anos, na pré-menopausa, com história de DM tipo 2 e hipertensão arterial sistêmica, em uso intermitente de Estatinas e Atorvastatina. Apresentou no exame físico massas indolores únicas e bilaterais nas mamas, sem linfadenomegalia, IMC de $28,2 \mathrm{~kg} / \mathrm{m}^{2}$, com circunferência abdominal elevada, Pressão Arterial (PA) de 120x180 mmHg e Frequência Cardíaca (FC) de 84 bpm, além de apresentar discreta hepatomegalia. Foi solicitada mamografia, a qual indicou múltiplos cistos com diâmetro máximo de 100mm e 4x13mm, massas heterogêneas, hipoecóicas na região 
subareolar da mama esquerda com aspecto vascularizado, caracterizando Bi-rads 4, sendo indicado realizar core-biopsy. A partir dessa, encontrou-se proliferação de histiócitos, sinal de mastopatia linfocítica com presença de células gigantes, atrofia lobular e inflamação linfocítica periductal. Ainda se verificou expressão de CD68 nos histiócitos e CD3 na população linfocítica. Exames laboratoriais sem achados de doenças autoimunes e de alterações significativas, exceto padrões analisados para DM. Dessa forma, os achados foram consistentes com mastopatia linfocítica/MD.

Ademais, Francisco et al. (2012) abordou caso de paciente do sexo feminino, de 34 anos, com histórico de DM tipo 1 insulino-dependente há 12 anos, retinopatia diabética e massas bilaterais nas mamas, indolores, mas sensíveis, tendo no exame físico nódulos duros, bilaterais, irregulares, indolores, nos quadrantes superiores, sem corrimento dos mamilos, sem anormalidades na pele e sem linfonodopatia axilar. A mamografia mostrou parênquima denso bilateralmente, BI RADS 4, com nódulos padrão, no quadrante superior, sem suspeita de microcalcificações, massas ou distorções na arquitetura, já a ressonância magnética detectou sinal heterogêneo de baixa intensidade e após administração de gadolíneo mostrou aprimoramento pobre e difuso sem aprimoramento focal ou nodular suspeito. A análise histológica da biópsia revelou estroma fibroso denso e infiltrado linfocítico periductal sem evidência ou atipia ou sugestão de malignidade. Visto isso, a MD é um diagnóstico diferencial de câncer de mama e, embora não seja uma doença pré-maligna, requer consciência da existência dessa condição, associada a uma análise cuidadosa do histórico clínico com exames físico, radiológicos e patológicos.

Em relato de caso publicado por Miura et al. (2012) abordou o primeiro relato de MD com autoanticorpos contra insulina e células ductais com reatividade cruzada, ao apresentar paciente de 74 anos, com histórico de DM insulinodependente há 31 anos, com massa na mama direita há 4 anos. Ao exame físico detectou-se a presença de dois nódulos endurecidos na mama direita, nenhum nódulo palpável na mama esquerda, ausência de alterações da pele adjacente e linfonodos axilares não palpáveis. Na mamografia somente a mama direita demonstrou uma densa massa microlobulada de 16 $\times 21 \mathrm{~mm}$ e uma pequena calcificação redonda à $43 \mathrm{~mm}$ do mamilo e no US apresentou área hipoecóica irregular com sombra acústica na posição de 9 a 12 horas e massa hipoecóica bem definida com sombra acústica posterior às 2 horas. Além disso, a mama esquerda apresentou área hipoecóica irregular com sombra acústica posterior às 2,5 horas. Com a realização de uma ressonância magnética observou-se massas microlobuladas densas com margens irregulares, com $43 \times 52 \times 37 \mathrm{~mm}$ e $17 \times 23 \times$ $14 \mathrm{~mm}$, ambas em mama direita, e também uma massa com características semelhantes de $11 \times 20 \times 15 \mathrm{~mm}$ em mama esquerda. Na citologia, realizada a partir da biópsia, foi possível visualizar a presença de células ductais e pequenos linfócitos, sugerindo linfoma maligno. A partir da excisão de massa à direita e sua análise macroscópica foi observada dimensões de $2,5 \times$ $2 \times 1 \mathrm{~cm}$ e malha composta por fibras esbranquiçadas separando o tecido gorduroso atrófico da mama, presença de pequenos linfócitos infiltrados ao redor dos ductos com densas fibras de colágeno circundantes, acompanhados por folículos linfáticos com centros germinativos que consistiam mais em células positivas para CD20 do que em células positivas para CD3. Visto isso, tem-se que o mimetismo molecular é caracterizado por uma resposta imune a um agente ambiental que reage de forma cruzada com um antígeno hospedeiro, resultando em doença, sendo essa causada pela DM.

Ainda, Nasu et al (2012), por sua vez, buscou evidenciar os achados presentes na Ressonância magnética (com e sem contraste) e no estudo anatomopatológico em duas pacientes com MD. Em seu estudo, ficou concluído que apesar de a MD ser composta de achados como tecido fibroso denso com pouca celularidade e de hipointensidade na DWI, em casos em que se tem marcadores de tecido infiltrativo e linfocítico, a hiperintensidade pode ser encontrada, se parecendo com tumores malignos de mama, sendo essencial a utilização de critérios consolidados. A primeira paciente, de 74 anos, pós-menopausa e com retinopatia diabética, apresentava duas massas palpáveis e duras nos quadrantes superiores mediais e quadrantes superiores laterais da mama direita há 4 meses. Ao realizar-se a RM, após a mamografia e o US, achou-se uma massa redonda e microlobulada no quadrante superior medial e uma massa irregular e mal definida no quadrante superior lateral da mama direita. As massas sofreram realce com o passar do tempo e após o contraste. Após tal exame, realizou-se a core biopsy, que 
identificou-se tecido fibroso com marcadores de infiltração linfocítica e suspeitou-se de MD. A massa do quadrante superior medial da mama direita foi retirada por resultado inconclusivo (após a cirurgia a análise verificou mastite com infiltração linfocítica e fibrose do estroma), com confirmação de MD. A segunda paciente, de 33 anos na pré-menopausa, também com retinopatia diabética e em uso de insulina há 18 anos, apresentava massa palpável e sem margens bem definidas no quadrante inferior lateral da mama esquerda, sem presença de mudança de pele e sem descarga mamilar. Após a mamografia e o US, realizou-se a RM, que em T2 mostrou uma massa hipointensa e de margens irregulares. Pós contraste teve-se realce heterogêneo e de pouca intensidade, aumentando com o passar do tempo. Realizou-se, assim, a core-biopsy, a qual identificou tecido fibroso com marcadores de infiltração linfocítica e suspeitou-se de MD, com confirmação diagnóstica.

Em estudo de Sankaye e Kachewar (2012), o objetivo foi relatar o caso de uma paciente de 52 anos, com histórico de DM tipo 1 insulino-dependente, sem histórico familiar de neoplasias, apresentando uma massa palpável, indolor em mama direita. No exame físico foi observado nódulo palpável, irregular e móvel na mama direita, sem alteração na pele adjacente e sem linfonodos axilares palpáveis. A mamografia apresentou parênquima mamário denso heterogêneo e presença de nódulo suspeito de malignidade. US revelou na mama direita lesão irregular de ecogenicidade mista medindo 3,4 x $2,5 \mathrm{~cm}$ e sombra acústica posterior. Foi realizada uma biópsia excisional cuja avaliação histológica mostrou lesão compatível com fibrose estromal e infiltração linfocítica periductal, sem qualquer atipia ou indicativo de malignidade. Portanto, foi concluído que um manejo clínico conservador e um acompanhamento rigoroso devem ser considerados sempre que houver característica clínicocitológicas consistentes com mastopatia diabética. Além disso, o conhecimento desta entidade rara e a correlação clínicoimagiológica-patológica no cenário clínico de diabetes mellitus ajuda a identificar esta condição e evita biópsias cirúrgicas desnecessárias, sofrimento mental, bem como incerteza diagnóstica.

A partir de sua pesquisa, Accurso et al. (2014), relatou caso incomum de MD, sendo paciente do sexo feminino, 61 anos, com histórico de DM2 há 8 anos, com complicações microvasculares e macrovasculares decorrentes da própria doença, e tireoidite de Hashimoto em tratamento com levotiroxina. Ao exame físico, apresentou massa endurecida em quadrante superior lateral em mama direita, sendo solicitados mamografia, US e aspirado por agulha fina. Como resultados, na mamografia foi indicada uma densidade localizada aumentada, com distorção de arquitetura com margens espiculadas, sugestivo de malignidade, em correspondência à massa palpável, e também houve essa distorção semelhante a nível dos quadrantes laterais, mas sem massa palpável. Ainda foram encontradas calcificações distróficas em ambas as mamas, principalmente na mama direita, mas sem relevância clínica. No US, evidenciou massa hipoecoica, de 10x82mm de diâmetro, sem margens definidas e com presença de sombra acústica posterior na parte superior do quadrante superior lateral direito, e em planos profundos encontrou-se outra lesão de características semelhantes, medindo 12x11mm. Já na aspiração por agulha fina, indicou linfócitos misturados a histiócitos. Entretanto, as duas massas foram ressecadas e enviadas para análise histopatológica, a qual apontou conteúdo fibrótico com estroma e infiltrado linfocítico importante, sendo confirmado o diagnóstico de MD. Sendo assim, mais uma vez faz-se necessário considerar MD como diagnóstico diferencial ao encontrar massa em mamas de pacientes com histórico de DM tipo 1 ou 2, sendo o diagnóstico histopatológico mandatório. Por fim, não há evidências de que a MD predisponha ao desenvolvimento de câncer de mama ou neoplasia de estroma, logo, deve-se evitar a cirurgia, uma vez que tem grandes chances de reincidência.

Além disso, de acordo com Leroux-Stewart e Rabasa-Lhoret (2014), foi feito um trabalho relatando o caso de uma paciente de 34 anos com histórico de diabetes mellitus tipo 1 insulino-dependente desde os 12 anos de idade e retinopatia diabética diagnosticada há 2 anos. No exame físico foi detectada uma massa endurecida, indolor, de cerca de $2 \mathrm{~cm}$ na mama esquerda. US revelou zona hipoecóica de 1,9 × 1,4 cm e na mamografia foi observado parênquima mamário extremamente denso sem lesão suspeita. $\mathrm{O}$ resultado da biópsia guiada por ultrassom apresentou fibrose semelhante a quelóide sem qualquer evidência de atipia, o que é compatível com o diagnóstico benigno de MD. Logo, entende-se que é uma doença rara e benigna, 
observada principalmente em mulheres com DM tipo 1, cujo reconhecimento é crucial para se evitar cirurgias e procedimentos desnecessários.

Outrossim, Moschetta et al. (2014) avaliou de forma retrospectiva a incidência e as características morfológicas da MD em um grupo de pacientes com diabetes, procurando por características ultrassonográficas específicas dessa doença. Nos arquivos eletrônicos da Universidade Aldo Moro da Escola Médica de Bari foram analisados os casos de 2340 pacientes, sendo que 1640 foram submetidas a mamografia e 2340 a ultrassonografia. Realizou-se também uma busca a partir das palavras chave "diabetes", "mastopatia diabética" e "doença diabética". Todos os casos com lesões nas mamas foram confirmados histologicamente e nas massa sólidas realizou-se biópsia com agulha grossa guiada por ultrassom, de modo que os resultados foram classificados segundo as diretrizes da FONCAM. Do total, 120 mulheres apresentaram diabetes (54\% diabetes tipo $1 \mathrm{e}$ 46\% diabetes tipo 2). Dessas, 11 apresentaram lesões nas mamas, das quais 9 correspondiam a MD e 2, que possuíam DM tipo 2, a carcinoma ductal invasivo com metástases em linfonodos axilares. Entre as pacientes com MD, 7 apresentavam massas duras com área densa, fibroglandular e indeterminada (na mamografia) de características sólidas hipoecóicas com margens irregulares, ecotextura homogênea e sombra posterior marcada (no US). A biópsia concluiu MD classificando as massas na categoria B2. Em 2 das pacientes com MD as massas não eram duras e a mamografia mostrava uma estrutura mamária muito densa, sem evidência de distorção glandular ou opacidade e no US apareceram massas sólidas hipoecóicas ligeiramente não homogêneas. Sendo assim, embora a MD seja rara, deve ser suspeitada em todos os pacientes portadores de DM e nódulo na mama. Os recursos de imagem, porém, são inespecíficos e altamente sugestivos na maioria dos casos, sendo a biópsia por agulha grossa obrigatória para fazer um diagnóstico definitivo.

Aja e seus colaboradores (2016), discutiram o uso da mamografia espectral de contraste aprimorado (CESM) a fim de identificar se essa técnica permite diferenciar lesões malignas de MD. Relatou caso de paciente do sexo feminino, de 35 anos, com histórico de DM tipo 1 insulino-dependente há 20 anos, sem histórico de malignidade na família, com nódulo palpável móvel na mama esquerda. Aos exames, US indicou lesão ecóica pouco definida, com $60 \mathrm{~mm}$ de diâmetro transversal e sombra acústica distal em quadrante lateral em relação à lesão, CESM demonstrou aumento da densidade do parênquima em quadrante superior lateral em mama esquerda e, de acordo com parâmetros, indicou área sugestiva de malignidade, sendo necessária confirmação com biópsia por agulha. A histopatologia dessa revelou estroma fibrótico com fibroblastos epitelióides, sem sinais de atipia, com presença de infiltrado linfocítico perivascular, sendo, compatível com MD. Desse modo, embora a CESM seja uma avanço na sensibilidade do diagnóstico de câncer de mama, neste caso o exame não permitiu a diferenciação entre MD e lesões malignas, o que não excluiu a necessidade de se realizar a biópsia para se confirmar o diagnóstico, algo que pretendia ser evitado.

O estudo de Gupta et al (2019), foi realizado com o objetivo de evidenciar os critérios diagnósticos e histológicos para a Mastopatia diabética e sua diferenciação com o CA de mama. Apresentou-se uma paciente com DM tipo 1 e com retinopatia diabética, doença vascular, doença renal em Hemodiálise, apresentando Hemoglobina Glicada de 7,1\% e em uso de insulina de longa duração. A mamografia mostrou a presença de tecido heterogêneo e denso em ambas as mamas, já o US demonstrou uma massa grande $(5.3 \times 1.9 \times 2.0 \mathrm{~cm})$ na mama direita, às 12 horas, com aspecto irregular, hipoecóico, com sombreamento acústico e densa vascularização periférica. Por se tratar de um Bi-rads 5, sugestivo de alta malignidade, realizou-se core biopsy guiada por US, que revelou Esclerose Linfocítica Lobular com tecido fibrocístico não proliferativo (sinal de inflamação no tecido mamário) e Mastite Linfocítica. Fez-se então diagnóstico de MD. Concluiu-se, assim, que a MD, uma doença fibrosa benigna e incomum, deve ser considerada como diagnóstico diferencial para achados nas mamas de pacientes com Diabetes, entretanto, é essencial a realização da análise histopatológica e de um diagnóstico com maior critério.

Em estudo de Alkhudairi et al (2020), buscou realizar o diagnóstico de MD a partir da clínica, da radiologia e da histopatologia a fim de amenizar a ansiedade do paciente e não realizar procedimentos desnecessários. Para isso, foi analisada, 
no período de 2020, uma paciente do sexo feminino, 29 anos, com nódulo único e indolor em mama esquerda, de aparecimento recente, de tamanho $5,4 \times 1,9 \mathrm{~cm}$, circunscrito, retroareolar, não aderido a planos profundos e sem alterações em pele adjacentes . Apresentava também histórico de DM tipo 1 há 18 anos, sendo insulino-dependente, mas sem complicações secundárias e com histórico familiar de câncer de mama. Foi realizada mamografia de mama esquerda, com vistas craniocaudal e mediolateral oblíqua, com típica representação de MD, incluindo parênquima mamário denso, assimetrias glandulares e sem lesões discretas identificáveis. Ademais, também foi feita uma biópsia guiada por US revelou tecido acelular fibroesclerótico com lóbulos e ductos de aparência normal, circundado por infiltrado linfocítico denso, sem evidências de malignidade, sendo estabelecido o diagnóstico de mastopatia diabética. Ainda assim, foi feita biópsia excisional que indicou intensa fibrose com colágeno e infiltrado linfocítico, sem atipias acelulares. Dessa forma, foi possível concluir que, apesar de MD ser menos prevalente que câncer de mama, deve-se atentar aos pacientes com longo histórico de MD que apresentam massa em mamas, já que se for diagnosticada, a abordagem leva em consideração sua natureza benigna, a fim de submeter o paciente a menos procedimentos invasivos.

\section{Considerações Finais}

A mastopatia diabética é, portanto, um importante diagnóstico diferencial para neoplasias de mama cujo diagnóstico é bastante complexo e exige, para além dos aspectos clínicos, a análise de componentes radiológicos e laboratoriais. As evidências sugerem que tanto a clínica, em que a mastopatia diabética se apresenta como massas palpáveis, quase sempre duras, não sensíveis e móveis, quanto nos exames de ultrassonografia e mamografia, as características são muito similares às neoplasias. Nesse sentido, a biópsia torna-se um exame fundamental à medida que os demais métodos diagnósticos se mostram inespecíficos, embora a história clínica de DM seja preditiva de mastopatia diabética. Além disso, vale ressaltar que a biópsia por agulha grossa se mostrou mais eficiente que a por agulha fina, uma vez que apresenta maiores probabilidades de se coletar a quantidade suficiente de material para uma adequada análise histopatológica.

Dessa forma, as perspectivas futuras envolvem ainda a utilização da biópsia para confirmação diagnóstica, embora seja um método invasivo, a menos que haja o desenvolvimento de algum exame de imagem que permita identificar particularidades da mastopatia diabética. Além disso, espera-se que os casos de mastopatia diabética tornem-se ainda mais comuns, visto que esta enfermidade pode desenvolver-se também em pacientes com diabetes mellitus tipo 2 , a qual é cada vez mais frequente devido aos hábitos alimentares e de sedentarismo da população.

\section{Referências}

Abdullah, S. S., et al. (2020). Mastopathy in a Patient with High Risk of Breast Carcinoma: A Management Dilemma. Cureus. 12 (2).

Accurso, A., et al. (2014). Unusual breast lesion mimicking cancer: Diabetic mastopathy. International Journal of Surgery. 12 (1).

Agochukwu, N. B., et al. (2017). Diabetic Mastopathy. Annals of Plastic Surgery. 4(78): 471-5.

Aja, M. M., et al. (2016). Is contrast-enhanced spectral mammography (CESM) helpful in differentiating diabetic mastopathy from breast carcinoma? Journal of Medical Imaging and Radiation Oncology. 60 (5): 639-41.

Alkhudairi, S., et al. (2020). Diabetic Mastopathy in a Patient with High Risk of Breast Carcinoma: A Management Dilemma. Cureus. 12 (2).

Bilir, B., et al. (2012). A Metabolic Syndrome Case Presenting with Lymphocytic Mastitis. Breast care. 7: $493-495$.

Casarin, S. T., et al. (2020). Tipos de revisão de literatura: considerações das editoras do Journal of Nursing and Health. J. nurs. health. 10 (5).

De Sousa, L. D., et al. (2011). The nursing scientific production about the clinic: an integrative review. Rev Esc Enferm, 45 (2), $494-500$.

Dorkhova, O., et al. (2012). Diabetic mastopathy, a clinicopathological correlation of 34 cases. Pathol Int. 62: 660-664.

Francisco, C., et al. (2012). Diabetic mastopathy: a case report. Clinical Imaging. 36: 829-832. 
Research, Society and Development, v. 10, n. 14, e260101421961, 2021

(CC BY 4.0) | ISSN 2525-3409 | DOI: http://dx.doi.org/10.33448/rsd-v10i14.21961

Gupta, S., et al. (2019). Diabetic Masthopaty Masquerading as Breast Cancer. The American Journal of Medicine. 132 (4).

Leroux-Stewart, J., \& Rabasa-Lhoret, R. (2014). Diabetic Mastopathy: Case Report and Summary of Literature. Canadian Journal of Diabetes. 38 (5): $305-$ 306.

Loscos, et al. Mastopatía Diabética. (2021). Revisión sistemática con 93 pacientes. Revista de Senología y Patología Mamaria. 34: 163 -170.

Machado, M. J. C., \& Regalo, G. (2020). Lymphocytic Mastopathy - A Rare Entity and A Diagnostic Challenge. International Journal of Surgical Reports. 2 (1).

Miura, K., et al. (2012). Autoantibody with Cross-Reactivity between Insulin and Ductal Cells May Cause Diabetic Mastopathy: A Case Study. Case Reports in Medicine. 2012.

Moschetta, M., et al. (2015). Diabetic Mastopathy: A Diagnostic Challenge in Breast Sonography. Journal of Clinical Ultrasound. 43: 2.

Nasu, H., et al. (2012). Two cases of diabetic mastopathy: MR imaging and pathological correlation. Breast Cancer. 22 (5).

Neetu, G., Pathmanathan, R., \& Weng, N. K. (2010). Diabetic mastopathy: a case report and literature review. Case Rep Oncol. 3: $245-51$.

Pereira, M. A., et al. (2010). Fibrous mastopathy: clinical, imaging, and histopathologic findings of 31 cases. J ObstetGynaecol Res. 36: 326-335.

Perret, W., Malara, F. A., Hill, P. A., \& Cawson, J. N. (2006). Painful Diabetic Mastopathy as a Reason for Mastectomy. The Breast Journal. 12 (6): 559-562.

Rajasundaram, S., et al. (2020). Diabetic mastopathy-An uncommon presentation of a common disease. The Breast Journal. 2020.

Sankaye, S., \& Kachewar, S. (2012). Diabetic mastopathy. Australasian Medical Journal. 6: 296-299.

Seidman., et al. (1994). Mastopathy in insulin-requiring diabetes mellitus. Hum Pathol. 5 (8).

Shrikrishnapalasuriya. ,et al. (2018). Diabetic mastopathy: a diagnostic challenge. Br J Diabetes. 18.

Soler, N. G., \& Khardori, R. (1984). Fibrous disease of the breast, thyroiditis, and cheiroarthropathy in type I diabetes mellitus. Lancet. 1 (8370): 193-5.

Tomaszewski, J. E., Brooks, J. S. J., Hicks, D., \& Livolsi, V. A. (1992). Diabetic mastopathy: A distinctive clinicopathologic entity. Human Pathology. 23 (7): $780-786$

Zhang, Y., et al. (2011). Diabetic Mastopathy Mimicking Breast Cancer: Two Case Reports. Clinical Breast Cancer. 11(6):409-12

Yamashita, M., et al. (2013). An uncommon case of T1b breast cancer with diabetic mastophaty in type II diabetes mellitus. Breast Cancer. 20 : 92-96. 